\title{
Incidência da pneumonia associada à ventilação mecânica em unidade de terapia intensiva
}

\author{
Incidence of ventilator-associated pneumonia in intensive care unit
}

Écila C. Mota ${ }^{1}$; Silvânia P. Oliveira²; Beatriz R. M. Silveira ${ }^{3}$; Patrick L. N. Silva ${ }^{4}$; Adriana C. Oliveira ${ }^{5}$

\begin{abstract}
RESUMO
Objetivo: avaliar a incidência da pneumonia associada à ventilação mecânica em unidade de terapia intensiva (UTI). Método: trata-se de um estudo de coorte retrospectivo desenvolvido em um hospital universitário. Análises univariadas foram realizadas por meio do teste Qui-quadrado ou teste exato de Fisher para variáveis categóricas e teste não paramétrico de Mann-Whitney para variáveis numéricas. Resultados: Durante 24 meses foram admitidos 190 pacientes na UTI, desses, 90,5\% utilizaram Ventilação Mecânica (VM). A incidência de pneumonia associada à VM foi de 23,2\%, sendo notificada em $100 \%$ dos pacientes que utilizaram VM; a densidade de incidência foi de 32,4/1000 ventiladores/dia e a taxa de mortalidade global dos pacientes com pneumonia foi de $72,7 \%$. Houve associação positiva entre a ocorrência de pneumonia e o tempo de internação >15 dias (RR: 7,29), o tempo de VM >10 dias (RR: 11,33 ) e reintubação (RR: 6,31). Conclusão: a pneumonia foi considerada como uma doença de alta morbidade na Unidade de Terapia Intensiva. Torna-se necessário a implantação de medidas efetivas para qualidade e segurança no cuidado de pacientes críticos.
\end{abstract}

Palavras-chave: Pneumonia Associada à Ventilação Mecânica. Unidades de Terapia Intensiva. Infecção Hospitalar. Segurança do Paciente.

\begin{abstract}
Objective: To evaluate the incidence of ventilator-associated pneumonia in intensive care unit (ICU). Method: A retrospective cohort study, was carried in a university hospital. Univariate analyzes were performed using the chi-square test or Fisher's exact test for categorical variables, and nonparametric Mann-Whitney test for numerical variables. Results: In a period of 24 months, 190 patients were admitted in the ICU, and $90.5 \%$ of them used mechanical ventilation. The incidence of ventilatorassociated pneumonia (VAP) was $23.2 \%$, being notified in $100 \%$ of the patients who used the mechanical ventilation; the incidence density of VAP was $32.4 / 1000$ ventilator days and the overall mortality associated with VAP was $72.7 \%$. There was a positive association between the occurrence of pneumonia and the period of hospitalization in the intensive care unit > 15 days (RR: 7.29), time of mechanical ventilation $>10$ days (RR: 11.33), and reintubation (RR: 6.31). Conclusion: pneumonia is considered a high morbidity condition in the intensive care unit. Thus, it is necessary to implement effective measures for quality and safety on the care of critically ill patients.
\end{abstract}

Key-words: Pneumonia, Ventilator-Associated. Intensive Care Units. Cross Infection. Patient safety.

1. Enfermeira, Doutoranda em Enfermagem, Universidade Federal de Minas Gerais (UFMG), Belo Horizonte, Brasil.

2. Enfermeira, Universidade Estadual de Montes Claros (UNIMONTES). Montes Claros, MG, Brasil.

3. Enfermeira, Mestre em Saúde Pública, UNIMONTES

4. Enfermeiro, especialista em Saúde da Família, UNIMONTES

5. Enfermeira, Doutora. Professor Associado da Escola de Enfermagem, UFMG.
CORRESPONDÊNCIA: Écila C. Mota

Escola de Enfermagem da UFMG. Av. Alfredo Balena 190, sala 200, Santa Efigênia. CEP: 30130100. - Belo Horizonte, MG. 


\section{Introdução}

A pneumonia hospitalar é definida como aquela que ocorre a partir de 48 horas da admissão do paciente ${ }^{1}$ e a segunda principal Infecção Relacionada à Assistência à Saúde (IRAS) em Unidade de Terapia Intensiva (UTI). Frequentemente estar associada ao uso da ventilação mecânica (VM) sendo denominada Pneumonia Associada à Ventilação Mecânica (PAVM). Trata-se assim da complicação infecciosa mais prevalente em terapia intensiva, com taxas que variam de $9 \%$ a $40 \%$ das IRAS adquiridas nessa unidade. A PAVM está associada a um aumento no período de hospitalização e descrevemse índices de mortalidade que podem variar de $24 \%$ a $76 \%$, repercutindo de maneira significativa nos custos hospitalares. . $^{1,2,3}$

A tecnologia altamente especializada e complexa utilizada em UTI incrementa a sobrevida dos pacientes críticos nas mais diversas situações, em contrapartida aumenta os fatores de riscos predisponentes que levam os pacientes internados a adquirirem IRAS, principalmente a PAVM. Dentre os fatores de risco, são reportados o uso indiscriminado de antimicrobiano prévio, a gravidade da doença de base, tempo de permanência prévio do paciente em outras unidades, ou em outras instituições de saúde, o tempo de permanência na UTI, os procedimentos invasivos a que são submetidos, como cateteres venosos centrais, cateterismo vesical e VM. ${ }^{4,5}$

A presença de tubo endotraqueal é apontada como um importante fator de risco para PAVM, especialmente por prejudicar as defesas do hospedeiro e permitir que partículas inaladas tenham acesso direto às vias aéreas inferiores. Além disso, a microbiota da cavidade bucal é uma ameaça aos pacientes em VM, pois a presença do tubo endotraqueal e o estado de inconsciência do paciente comprometem a realização da higiene bucal, favorecendo, ainda mais, a proliferação microbiana. ${ }^{6-9}$

Os fatores de risco para o desenvolvimento de pneumonia hospitalar podem ser classificados em modificáveis ou não-modificáveis. Os fatores não-modificáveis são: idade elevada, maior escore de gravidade do paciente no momento da internação, doença pulmonar obstrutiva crônica (DPOC), doença neurológica, trauma e cirurgia. Por outro lado, os fatores modificáveis incluem o tempo de duração da VM, reintubação, traqueostomia, uso de sonda nasogástrica (SNG) ou sonda nasoentérica (SNE), uso de dieta enteral, aspiração de conteúdo gástrico, uso prévio de antimicrobianos, e a permanência em posição supina. ${ }^{1}$

A PAVM é uma infecção comum na UTI, mas seu diagnóstico permanece desafiador. O diagnóstico é feito quando o paciente desenvolve um novo infiltrado pulmonar ao exame de imagem acompanhado pela presença de febre, leucocitose e secreção traqueal purulenta. A utilização desses critérios para diagnosticar a PAVM apresenta alta sensibilidade, porém baixa especificidade, pois a febre pode ser causada por reação medicamentosa ou outra infecção extrapulmonar, e os infiltrados pulmonares podem ser decorrentes de derrame não infectado, aspiração química, ou outras causas. Diante disso, torna-se necessário a execução dos exames microbiológicos por meio da coleta de amostras de material do trato respiratório inferior, com a realização de culturas quantitativas no intuito de estabelecer um diagnóstico mais preciso. ${ }^{9}$

Nessa perspectiva, diante da relevância do tema no contexto das IRAS e sua importância epidemiológica, este estudo propõe investigar a seguinte questão de pesquisa: Qual a incidência de PAVM em uma UTI de um hospital universitário no norte de Minas Gerais (MG)?

Objetivou-se determinar a incidência de PAVM, bem como seus fatores de risco, agentes etiológicos e evolução dos pacientes em uma UTI de um hospital universitário no norte de Minas Gerais (MG).

\section{Métodos}

Trata-se de um estudo de coorte retrospectivo, desenvolvido em um Hospital Universitário no norte de Minas Gerais (MG) que possui 186 leitos, exclusivamente conveniados ao Sistema Único de Saúde (SUS), sendo desses seis de UTI adulto. A unidade é classificada como geral, atende tanto pacientes clínicos como cirúrgicos e possui taxa de ocupação de $90 \%$. O hospital possui Serviço de Controle de Infecção Hospitalar (SCIH) atuante e não utiliza o pacote de medidas (bundles) para prevenção da PAVM. Os dados foram coletados por meio de um instrumento semiestruturado, adaptado e testado pelo protocolo de PAVM do SCIH da instituição, contendo variáveis relacionadas ao sexo, 
idade, clínica de internação prévia, utilização e tempo de VM, reintubação, diagnóstico de outras infecções, tempo de internação na UTI, resultados de culturas e antibiogramas e evolução/desfecho do paciente (alta, óbito ou transferência).

A coleta de dados foi realizada por meio da análise dos prontuários de todos os pacientes internados na UTI, disponibilizados pelo Serviço de Arquivo Médico e Estatístico (SAME). Utilizou-se também, as fichas de notificações de IRAS do SCIH para conferência dos dados. Foram incluídos na pesquisa 190 pacientes adultos internados na UTI, no período de janeiro de 2011 a dezembro de 2012 . Os dados e a lista referentes ao total de pacientes internados foram fornecidos pelo SAME do hospital. Não houve perda da população, todos os prontuários foram analisados desde a admissão até a alta. A variável dependente foi à ocorrência de PAVM e, como variáveis independentes dados referentes ao sexo, idade, diagnóstico na admissão, uso e tempo de VM, reintubação, dieta enteral, antibioticoterapia prévia, resultados de cultura, germes, topografia de IRAS e evolução/desfecho do paciente.

Os critérios utilizados para definição da PAVM foram os estabelecidos pela Agência Nacional de Vigilância Sanitária (ANVISA), e os mesmos são utilizados pelo SCIH do referido hospital. Foram considerados critérios radiológicos como: exame de imagem com infiltrado, opacificação, cavitação; Clínicos: febre, definida como temperatura axilar acima de $37,8^{\circ} \mathrm{C}$, surgimento e/ou aumento de secreção purulenta; e critérios laboratoriais: leucopenia ou leucocitose, hemograma com hemocultura e cultura do líquido pleural positiva, lavado broncoalveolar maior ou igual a $104 \mathrm{UFC/ml}$ ou aspirado traqueal com contagem de colônias maior ou igual a $106 \mathrm{UFC} / \mathrm{ml}$.

Inicialmente foi feita uma análise descritiva de todas as variáveis do estudo apresentadas por meio de tabelas com distribuição de frequência e medidas de tendência central e variabilidade. $\mathrm{Na}$ análise univariada, foi realizado o teste Qui-quadrado de Pearson ou teste exato de Fisher na comparação das variáveis categóricas. Para as variáveis numéricas optou-se pelo teste não-paramétrico de Mann-Whitney devido ao caráter assimétrico das variáveis testadas. A força de associação entre as variáveis independentes e a dependente foi expressa em estimativas de Risco Relativo (RR). Em todas as análises considerou-se um nível de significância de $5 \%$ e foi utilizado o software estatístico Statistical Package for Social Sciences (SPSS) versão 18.0.

A pesquisa obedeceu às normas da Resolução 466/2012 do Conselho Nacional de Saúde, ${ }^{10}$ que regulamenta pesquisas com seres humanos e foi aprovada pelo Comitê de Ética em Pesquisa da Universidade Estadual de Montes Claros (CEP UNIMONTES), sob parecer no 276.240.

\section{Resultados}

A população foi constituída de 190 pacientes adultos internados na UTI, no período de 24 meses, janeiro de 2011 a dezembro de 2012. A média de idade dos pacientes internados foi de 56,9 anos (desvio padrão de $\pm 18,6$ ). O tempo médio de internação foi de 9,5 \pm 16 dias, o tempo de VM até o diagnóstico de PAVM foi de 17,4 \pm 11.6 dias, a taxa de pneumonia foi de $23,2 \%$ e a densidade foi de 32,4/1000 VM (Tabela 1).

Tabela 1. Características sócio-demográficas e clínicas dos pacientes internados na UTI adulto. Montes Claros, 2011-2012

\begin{tabular}{|c|c|c|}
\hline Variáveis & $n$ & $\%$ \\
\hline \multicolumn{3}{|l|}{ Sexo } \\
\hline Feminino & 77 & 40,5 \\
\hline Masculino & 113 & 59,5 \\
\hline \multicolumn{3}{|l|}{ Faixa etária } \\
\hline Menor que 60 anos & 102 & 53,7 \\
\hline Maior que 60 anos & 88 & 46,3 \\
\hline \multicolumn{3}{|l|}{ Tempo de internação } \\
\hline Menor que 15 dias & 156 & 82,1 \\
\hline Maior que 15 dias & 34 & 17,9 \\
\hline \multicolumn{3}{|c|}{ Paciente transferido de outro local } \\
\hline Sim & 188 & 98,9 \\
\hline Não & 2 & 1,1 \\
\hline \multicolumn{3}{|l|}{ Utilização de VM } \\
\hline Sim & 172 & 90,5 \\
\hline Não & 18 & 9,5 \\
\hline \multicolumn{3}{|l|}{ Tempo de VM } \\
\hline Menor que 10 dias & 136 & 71,6 \\
\hline Maior que 10 dias & 54 & 28,4 \\
\hline \multicolumn{3}{|c|}{ Diagnóstico de pneumonia hospitalar } \\
\hline Sim & 44 & 23,2 \\
\hline Não & 146 & 76,8 \\
\hline \multicolumn{3}{|l|}{ Outras infecções } \\
\hline Sim & 18 & 9,5 \\
\hline Não & 172 & 90,5 \\
\hline
\end{tabular}


Diante das análises clínicas e laboratoriais relacionadas aos pacientes internados na UTI, observou-se uma taxa de reintubação de $12,6 \%$. No que diz respeito à terapia nutricional, $61,1 \%$ receberam dieta enteral. Para o diagnóstico de PAVM foi realizada hemocultura e aspirado/lavado traqueal em $48,4 \%$ dos pacientes, com resultado positivo em $28,4 \%$. Destaca-se que entre as culturas positivas, $16(29,6 \%)$ foram classificados como microrganismos multirresistente segundo Manual Clinical and Laboratory Standards Institute - CLSI, sendo cinco $(31,2 \%)$ Acinetobacter baumannii, quatro (25\%) Klesiella pneumoniae, quatro (25\%) Pseudomas aeruginosa, dois (12,5\%) Staphylococcus aureus e um (6,3\%) Escherichia coli. Quanto à antibioticoterapia, verificou-se que $26,3 \%$ dos pacientes fizeram uso prévio de antimicrobianos. A taxa de mortalidade geral na UTI foi de $84,7 \%$ ( $T a-$ bela 2).

A distribuição dos pacientes com ou sem diagnóstico de pneumonia estão apresentados na Tabela 3. Aqueles com tempo de VM maior de dez dias foram mais suscetíveis a desenvolver pneumonia (RR: $11,33, p<0,001$ ). Ainda, foi verificado como fatores de risco para o desenvolvimento de pneumonia nosocomial o uso da VM $(p<0,014)$; tempo de internação maior que 15 dias (RR:7,29, $p<0,001$ ), ocorrência de reintubação (RR: 6,31, $p<0,001)$ e diagnóstico de outras infecções (RR: $6,06, p<0,001$ ).

Os resultados das variáveis idade, tempo de internação e ventilação mecânica foram analisados no formato contínuo. Houve diferença estatística entre as médias $(p<0,001)$ do tempo de internação e VM dos pacientes que desenvolveram a pneumonia comparado àqueles sem a doença. Não foi observada associação significativa entre as médias de idades dos pacientes que desenvolveram pneumonia, comparados àqueles sem a doença $(p>0,05)$.

\section{Discussão}

A maioria dos pacientes deste estudo foram do sexo masculino e não houve diferença estatística significativa para PAVM entre gêneros e faixa etária. Estudos realizados em UTI de Campo Grande-MS e Rio Grande do Sul apontaram resultados semelhantes ao do presente estudo com taxa da população masculina de $55 \% .{ }^{11,12}$
Tabela 2. Análise descritiva das variáveis clínicas dos pacientes internados na UTI adulto. Montes Claros, 2011-2012.

Variáveis n $\quad \%$

\section{Reintubação na UTI}

Sim

$24 \quad 12,6$

Não

166

87,4

\section{Dieta enteral}

Sim

$116 \quad 61,1$

Não

$74 \quad 38,9$

Cultura aspirado traqueal

ou hemocultura

Sim

92

48,4

Não

$98 \quad 51,6$

Material da cultura

Aspirado/Lavado traqueal

Hemocultura

\section{Resultado aspirado traqueal ou} hemocultura

Positivo

Sem crescimento de micro-organismo

Micro-organismo isolado de aspirado

\section{traqueal ou hemocultura}

Staphylococcus aureus

Pseudomonas aeruginosa

Acinetobacter baumannii

Klebsiella pneumoniae

Enterobacter

Streptococcus $s p$

Escherichia Coli

Corynebacterium

Micro-orrganismo multirresistente isolado de aspirado traqueal ou

\section{hemocultura}

Sim

Não

Antibioticoterapia prévia à cultura

\section{Evolução do paciente}

Óbito

Transferência 
Tabela 3. Comparação da ocorrência de pneumonia hospitalar com os fatores sócio-demográficos e clínicos dos pacientes internados na UTI adulto. Montes Claros, 2011-2012.

\begin{tabular}{|c|c|c|c|c|c|c|}
\hline \multirow[b]{3}{*}{ Variáveis } & \multicolumn{4}{|c|}{ Pneumonia Hospitalar } & \multirow{3}{*}{$\begin{array}{c}\text { Risco Relativo } \\
\text { [IC 95\%] }\end{array}$} & \multirow[b]{3}{*}{ Valor-p } \\
\hline & \multicolumn{2}{|c|}{$\underset{(n=44)}{S i m}$} & \multicolumn{2}{|c|}{$\begin{array}{c}\text { Não } \\
(n=146)\end{array}$} & & \\
\hline & $n$ & $\%$ & $n$ & $\%$ & & \\
\hline \multicolumn{7}{|l|}{ Sexo } \\
\hline Feminino & 16 & 36,4 & 61 & 41,8 & 1,00 & \multirow{2}{*}{$0,521 *$} \\
\hline Masculino & 28 & 63,6 & 85 & 58,2 & $1,19[0,69-2,05]$ & \\
\hline \multicolumn{7}{|l|}{ Faixa etária } \\
\hline Menor que 60 anos & 22 & 50 & 80 & 54,8 & 1,00 & \multirow{2}{*}{$0,576 *$} \\
\hline Maior que 60 anos & 22 & 50 & 66 & 45,2 & $1,16[0,69-1,94]$ & \\
\hline \multicolumn{7}{|l|}{ Tempo de internação } \\
\hline Menor que 15 dias & 17 & 38,6 & 139 & 95,2 & 1,00 & \multirow{2}{*}{$<0,001^{*}$} \\
\hline Maior que 15 dias & 27 & 61,4 & 7 & 4,8 & $7,29[4,51-11,78]$ & \\
\hline \multicolumn{7}{|l|}{ Utilização de VM } \\
\hline Sim & 44 & 100 & 128 & 87,7 & ND & \multirow{2}{*}{$0,014^{*}$} \\
\hline Não & 0 & 0 & 18 & 12,3 & & \\
\hline \multicolumn{7}{|l|}{ Tempo de VM } \\
\hline Menor que 10 dias & 8 & 5,9 & 128 & 94,1 & 1,00 & \multirow{2}{*}{$<0,001 *$} \\
\hline Maior que 10 dias & 36 & 66,7 & 18 & 33,3 & $11,33[5,64-22,78]$ & \\
\hline \multicolumn{7}{|l|}{ Reintubação na UTI } \\
\hline Sim & 21 & 87,5 & 3 & 12,5 & \multirow{3}{*}{$\begin{array}{c}6,31[4,20-9,50] \\
1,00\end{array}$} & \multirow{3}{*}{$<0,001 *$} \\
\hline Não & 23 & 13,9 & 143 & 86,1 & & \\
\hline Não & 27 & 15,7 & 145 & 84,3 & & \\
\hline \multicolumn{7}{|l|}{ Evolução do paciente } \\
\hline Óbito & 32 & 72,7 & 129 & 88,4 & $2,08[1,22-3,55]$ & \multirow{2}{*}{$0,012 *$} \\
\hline Transferência & 12 & 27,3 & 17 & 11,6 & 1,00 & \\
\hline
\end{tabular}

*Teste Qui-Quadrado de Person

Nesta investigação, a incidência de PAVM, demonstrou uma taxa de $23,2 \%$, e todos os pacientes diagnosticados com pneumonia, utilizaram VM. Estudos realizados em hospital na Bahia, Santa Catarina, e região norte do Irã, corroboraram com os resultados dessa pesquisa, com taxa de incidência entre $22 \%$ a $26 \% .{ }^{13,14,15}$ Por mais que pacientes internados em UTI se beneficiem de cuidados multiprofissionais intensivos e altas tecnologias, eles são também submetidos a um elevado número de procedimentos e dispositivos invasivos, elevando assim o risco de adquirir infecções hospitalares (IH).
Autores ${ }^{15,16}$ reconhecem que, embora essas IH representem um desequilíbrio entre mecanismos de defesa e microbiota normal, comumente observada em pacientes gravemente doentes, a mesma pode também ser decorrente de determinadas condições de saúde responsáveis pela internação, ou procedimentos invasivos aplicados aos pacientes. Quando os pacientes intubados são submetido à VM, os mecanismos de defesa do pulmão estão alterados com perda da proteção das vias aéreas superiores e modificações da fisiologia normal respiratória o que contribui para o alto índice de morbida- 
de e mortalidade. De acordo com National Healthcare Safety Network (NHSN) (do Center of Disease Control and Prevention (CDC) Atlanta, USA, a PAVM representa a causa mais comum de IRAS em UTI. ${ }^{17}$ Essa afirmação vai ao encontro dos resultados obtidos de trabalhos realizados nos anos de 2011 e 2012 nos estados de Minas Gerais (MG) e Rio Grande do Sul (RS) respectivamente. ${ }^{8,18}$

No presente estudo, verificou-se que o uso de VM foi um fator desencadeante para a ocorrência de pneumonia hospitalar, pois o uso da VM foi aplicado em $100 \%$ dos pacientes diagnosticados com a doença. Resultados de pesquisa realizada na UTI de Campo Grande demonstraram que $83,8 \%$ dos pacientes que tiveram pneumonia hospitalar, fizeram uso de VM, o que mostra a relação significativa do uso de VM com a ocorrência de pneumonia hospitalar. ${ }^{19}$ É uma luta incansável a busca pela manutenção da vida dos pacientes críticos que necessitam de monitorização e suporte contínuo para preservação de suas funções vitais, na qual a maioria é submetida a procedimentos invasivos, como tubo orotraqueal, traqueostomia e VM, tendo como consequência a PAVM. ${ }^{7}$

Uma associação positiva foi identificada entre aqueles pacientes que usaram VM por mais de dez dias em relação à pneumonia (RR: 11,33$)$. Resultado semelhante foi observado em pesquisa realizada no Rio de Janeiro, que demonstrou que a presença de pneumonia com o tempo de $\mathrm{VM}$ foi fator de risco independente para o desenvolvimento de pneumonia. ${ }^{20}$ Estratégias para redução do tempo de VM devem ser usadas para diminuir o risco de PAVM, por isso é fundamental estabelecer em todas as UTI, protocolos direcionados ao uso racional de sedação e desmame.

Como prevenção, tem sido bastante utilizada os Pacotes de Cuidados ou bundle, os quais reúnem um pequeno grupo de intervenções que, quando implementadas em conjunto, resultam em melhorias substanciais na assistência em saúde, com possibilidade de redução da taxa de PAVM de 4,08 casos por 1000 ventilações dia para 1,6 casos por 1000 ventilações dia. ${ }^{21} \mathrm{O}$ bundle constitui um conjunto de práticas baseadas em evidências que, quando executadas coletivamente melhoram os resultados comparada ao uso de medidas individuais. O bundle de prevenção da PAVM, abrange quatro principais recomendações fundamentais: higiene das mãos e higiene oral com clorexidina $0,12 \%$; cabeceira elevada $30-45^{\circ}$; pressão do cuff entre $20-30 \mathrm{~cm} \mathrm{H}_{2} \mathrm{O}$; e cuidados com aspiração das secreções traqueais. ${ }^{5}$

No que se refere ao tempo de permanência na UTI, a população objeto do estudo que desenvolveu PAVM apresentou média de 26,2, semelhante a outros achados na literatura que mostram uma variação de 19,4 a 23,4 dias. Esses achados apontam para a importância do tempo de permanência na UTI, com maior risco para a ocorrência de infecção hospitalar. 8,15,22,23

A reintubação dos pacientes e ocorrência de pneumonia também esteve associada (RR: 6,31) de forma estatisticamente significativa. Trabalho realizado em uma UTI do Hospital de Clínicas da Universidade Estadual de Campinas aponta uma associação entre pacientes em relação à reintubação e tempo prolongado de permanência na UTI e reintubação e fatores de risco associado à mortalidade. ${ }^{13}$

O conhecimento dos fatores de risco é de fundamental importância para a tomada de decisão do controle e prevenção da doença. As Diretrizes Brasileiras de Pneumonia e Tisiologia ${ }^{1}$ afirmam que tempo de internação e VM, assim como reintubação, são fatores de risco para o desenvolvimento de pneumonia hospitalar.

No presente estudo, o uso da dieta enteral não foi relacionada ao risco para o diagnóstico de pneumonia, embora tenha sido para refluxos gastroesofágicos e aspiração de conteúdos colonizados no estômago ${ }^{24}$, incrementando a possibilidade de PAVM de forma indireta. ${ }^{22}$

Além de critérios clínicos, os pacientes sob suspeita de infecção devem se submeter a estudos microbiológicos. Os principais microrganismos envolvidos com a etiologia da pneumonia foram os gram-positivos seguidos pelas enterobactérias de forma semelhantes aos observados em um estudo no Rio de Janeiro. ${ }^{20}$ As PAVM, na maioria dos casos são provocadas por bactérias e em menor frequência, por vírus ou fungos. No entanto, o agente etiológico da PAVM depende do tipo de paciente, tempo de hospitalização, método de diagnóstico empregado e uso de terapia antimicrobiana. ${ }^{24}$

No tocante aos microrganismos isolados dos casos de pneumonia desse estudo, 29,6\% apresentaram um perfil de multirresistência antimicrobiana, diferentemente do estudo desenvolvido no norte do Brasil que detectou $54,5 \%$ de bactérias multirre- 
sistentes. ${ }^{25}$ Em uma pesquisa realizada para determinar o impacto dos microrganismos multirresistentes na morbimortalidade de pacientes com PAVM, - Staphylococcus aureus apareceu como o principal causador de PAVM, seguido do Pseudomonas aeruginosa. ${ }^{26}$

O fenômeno da resistência bacteriana está diretamente relacionado ao uso indiscriminado e inadequado dos antimicrobianos, somado à baixa adesão de protocolos e medidas de controle de infecção. ${ }^{26} \mathrm{~A}$ exposição prévia do paciente aos agentes antimicrobianos tem sido reconhecidamente associada a sua colonização bem como facilitador para o desenvolvimento da infecção. ${ }^{28}$ Reforçando que as infecções causadas por bactérias resistentes aos antibióticos nas UTI são precedidas pela colonização, a qual pode resultar da aquisição de microrganismo endógeno ou exógeno. ${ }^{29}$

Em relação à evolução do paciente verificouse que a maioria daqueles que desenvolveram PAVM evoluíram para óbito ainda que não tenha sido avaliada a mortalidade direta atribuída a PAVM. Embora essa análise direta não tenha sido realizada trabalho semelhante em UTI evidenciou mortalidade significativamente maior no grupo de pacientes com pneumonia hospitalar, reafirmando a PAVM como um fator de risco independente para a mortalidade na UTI. 20

\section{Conclusão}

Por meio deste estudo foi possível constatar que a incidência de PAVM foi de $23,2 \%$, como fatores de risco associado à sua ocorrência, identificaram-se o tempo de permanência na UTI, o uso de VM, o tempo de VM e a reintubação. Chamou atenção a elevada mortalidade em pacientes que desenvolveram a PAVM na UTI, ainda que não tenha sido analisada sua associação direta, no entanto não se pode desconsiderar o possível reflexo do perfil dos pacientes dessa unidade. Nesse contexto, medidas de prevenção de pneumonia em UTI devem ser implementadas com avaliação continuada sobre a sua ocorrência e tais desfechos, como por exemplo, a adoção de medidas, como o bundle, que podem seguramente contribuir para redução de sua incidência e promoção da qualidade e segurança no cuidado ao paciente sob assistência ventilatória invasiva.

\section{Referências}

1. Sociedade Brasileira de Pneumologia e Tisiologia. Diretrizes brasileiras para tratamento das pneumonias adquiridas no hospital e das associadas à ventilação mecânica 2007. J Bras Pneumol. 2007;33(Suppl 1):1-30.

2. Munro CL, Grap MJ, Jones DJ, Mcclish DK, Sesser CN. Chlorhexidine, toothbrushing, and preventing ventilatorassociated pneumonia in critically I'll adults. Am J Crit Care. 2009;18:428-37.

3. Pravin Charles MV, Kali A, Easow JM, Joseph NM, M Ravishankar, Srinivasan S, et al. Ventilator-associated pneumonia. Australas Med J. 2014; 7: 334-44.

4. Schwartzmann PV, Volpe GJ, Vilar FC, Moriguti JC. Pneumonia comunitária e pneumonia hospitalar em adultos. Medicina (Ribeirão Preto). 2010;43:238-48.

5. Rosenthal VD, Rodrigues C, Álvarez-Moreno C, Madani N, Mitrev Z, Ye G, et al. Effectiveness of a multidimensional approach for prevention of ventilator-associated pneumonia in adult intensive care units from 14 developing countries of four continents: findings of the International Nosocomial Infection Control Consortium. Crit Care Med. 2012;40:3121-8.

6. Damas P, Frippiat, F, Ancion A, Canivet J, Lambermont B, Layios N, et al. Prevention of ventilator-associated pneumonia and ventilator-associated conditions: a randomized controlled trial with subglottic secretion suctioning. Crit Care Med. 2015;43:22-30.

7. Bhat V, Kelkar R, Biswas S, Natarajan G. Ventilator-associated pneumonia: Survey of infection control practices in intensive care units of 15 tertiary care hospitals in Mumbai. Am J Infect Control. 2014;42:935-6.

8. Beraldo CC; Andrade D. Higiene bucal com clorexidina na prevenção de pneumonia associada à ventilação mecânica. J Bras Pneumol. 2008;34:707-14.

9. Ivor S, Douglas CS, Price $\mathrm{KH}$, Overdier RF, Wolken SW, Metzger KR, et al. Rapid Automated Microscopy for Microbiological Surveillance of Ventilator-associated Pneumonia. Am J Respir Crit Care Med. 2015;191:566-73.

10. BRASIL. Ministério da Saúde. Conselho Nacional de Saúde. Resolução no. 466, de 12 de dezembro de 2012. Homologa e regulamenta a realização de pesquisas envolvendo seres humanos nos termos do Decreto de Delegação de Competência de 12 de novembro de 1991.

11. Souza AS. Prevalência de pneumonia associada à assistência à saúde em unidades de terapia intensiva [Dissertação]. Universidade Federal de Mato Grosso de Sul, Campo Grande, 2012.

12. Favarin SS, Camponogara S. Perfil dos pacientes internados na unidade de terapia intensiva adulto de um hospital universitário. Rev Enferm UFSM. 2012;2:320-9.

13. Oliveira AC, Kovner CT, Silva RS. Infecção hospitalar em unidade de tratamento intensivo de um hospital universitário brasileiro Rev Latinoam Enferm. 2010;18:97-104.

14. Silva LTR, Laus AM, Canini SRMS, Hayashida M. Avaliação das medidas de prevenção e controle de pneumonia associada à ventilação. Rev Latinoam Enferm. 2011;19:1-9.

15. Lahoorpour F, Delpisheh A, Afkhamzade A. Risk factors for acquisition of ventilator-associated pneumonia in adult intensive care units. Pak J Med Sci. 2013;29:1105-7.

16. Keyt $H$, Faverio $P$, Restrepo MI. Prevention of ventilatorassociated pneumonia in the intensive care unit: A review of the clinically relevant recent advancements. Indian J Med Res. 2014;139:814-21.

17. The National Healthcare Safety Network (NHSN) Manual. Patient Safety Component Protocol. Centers for Disease Control and Prevention, January 2008. 
18. Moreira BSG, Silva RMO, Esquivel DN, Fernandes JD. Pneumonia associada à ventilação mecânica: medidas preventivas conhecidas pelo enfermeiro. Rev Baiana Enferm. 2011;25:99-106.

19. Souza AF, Guimarães AC, Ferreira EF. Avaliação da implementação de novo protocolo de higiene bucal em um centro de terapia intensiva para prevenção de pneumonia associada à ventilação mecânica. REME Rev Min Enferm. 2013;17:177-84.

20. Rodrigues PMA, Carmo NE, Santos LRC, Knibel MF. Pneumonia associada à ventilação mecânica: epidemiologia e impacto na evolução clínica de pacientes em uma unidade de terapia intensiva. J Bras Pneumol. 2009;35:108491.

21. Bezerra EL, Lima AIF, Nóbrega ARR, Barroso DN, Donadi HA, Santos JGS et al. Prevalência de pneumonia em pacientes de uma unidade de terapia intensiva de um hospital-escola de fortaleza - CE. Rev Bras Promoç Saúde. 2012;25(Supl. 2):20-4.

22. Freitas ERFS. Perfil e gravidade dos pacientes das unidades de terapia intensiva: aplicação prospectiva do escore APACHE II. Rev Latinoam Enferm. 2010;18:20-6.

23. Diaz MMO. Pneumonia associada à ventilação em pacientes internados nas unidades de terapia intensiva de hospital público e privada [Dissertação]. Universidade de Brasília, Brasília, 2011.
24. Martins SG, Pulcinelli RSR, Santos ARCV. Prevalência e perfil de resistência de microrganismos isolados do trato respiratório inferior de pacientes internados no Hospital Divina Providência. RBAC. 2008;40:83-6.

25. Resende MM, Monteiro SG, Callegari B, Figueiredo PMS, Monteiro CRAV, Neto VM. Epidemiology and outcomes of ventilator-associated pneumonia in northern Brazil: an analytical descriptive prospective cohort study. BMC Infect Dis. 2013;13:119.

26. Figueiredo DA, Vianna RPT, Nascimento JA. Epidemiologia da infecção hospitalar em uma unidade de terapia intensiva de um hospital público municipal de João Pessoa-PB. Rev Bras Ciênc Saúde. 2013;17:233-40.

27. Nóbrega MS, Carmo Filho JR, Pereira MS. Evolução da resistência de Pseudomonas aeruginosa e Acinetobacter baumannii em unidades de terapia intensiva. Rev Eletrônica Enferm. 2013;15:696-703.

28. Ribas RM, Gontijo Filho PP, Cezário RC, Silva PF, Langoni DRP, Duque AS. Fatores de risco para colonização por bactérias hospitalares multiresistentes em pacientes críticos, cirúrgicos e clínicos em um hospital universitário brasileiro. Rev Med Minas Gerais. 2009;19:193-7. 